\title{
Pump-Probe and Time-Resolved Electron Microscopy and Big Data Analysis of Electron-Lattice Dynamics in Strongly Correlated Electron Systems
}

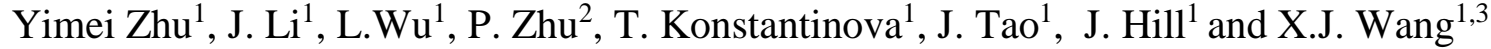 \\ 1. Brookhaven National Laboratory, Upton, NY USA \\ ${ }^{2}$ Shanghai Jiaotong University, Shanghai, China \\ ${ }^{3}$ SLAC National Accelerator Laboratory, Menlo Park, CA USA
}

The recent development of ultrafast imaging and diffraction opened a new frontier for studying structural dynamics at nanoscales. It has become one of the key directions for future electron microscopy. Ultrafast electron microscopy combines the superior spatial resolution of conventional electron microscope with short electron pulses enabling the detection of electronic and atomic motion on their natural time and lengths scales. Two paths have been pursued by modifying commercial TEMs. One is the multi-shot UEM [1] utilizing the single electron limit (one or few electrons per pulse) that circumvents the repulsive space charge problem but requires multimillion-shot accumulation to get a decent image thus suitable for repeatable dynamic events. The other is the single-shot DTEM [2] to achieve nm- $\mu$ s resolution. In parallel home-built ultrafast electron diffraction (UED) instruments have also been developed, especially the RF-based UED using accelerator technology that enabled to developing x-ray free electron lasers. Among the state-of-the-art electron sources, only photocathode RF guns are capable of generating $>10^{7}$ electrons per pulse, multi-MeV energy and sub ps-long electron beams. The distinct merit of photocathode RF guns is the very high acceleration gradient $(>100 \mathrm{MV} / \mathrm{m})$, over two-order of magnitude higher than $50 \mathrm{KeV}$ DC-guns [3]. It enables to minimize considerably the space charge effects, which can be scaled as inversely proportion to the product of velocity square and energy cube of the electrons, thus yielding much higher current density and beam brightness as recently demonstrated at Brookhaven $[4,5]$.

One of the major challenges in ultrafast electron microscopy is the analysis of big data sets. To improve signalnoise ratio very often ten thousands of diffraction patterns or images have to be acquired and analyzed. In this talk we will present our method on quantitative data analysis for two case studies related to photoinduced structural dynamics of the charge-density wave (CDW) state in $2 \mathrm{H}-\mathrm{TaSe}_{2}$ [4] and the charge-orbital ordered state in bi-layered $\mathrm{LaSr}_{2} \mathrm{Mn}_{2} \mathrm{O}_{7}$ at $77 \mathrm{~K}$ using MeV-UED. By simultaneously tracking both the melting of the periodic lattice distortion (PLD) associated with the CDW and the lattice heating, following an impulsive photoexcitation, the separate contributions of electronic excitation and lattice thermalization to the melting process are disentangled in the time domain. Distinct time-constants, reflecting the corresponding individual dynamics of the electronic and lattice systems, are observed. Our results demonstrate, for the first time in $2 \mathrm{H}-\mathrm{TaSe}_{2}$, that the PLD is first suppressed promptly by the electronic excitation and scattering, and then subsequently by lattice thermalization through electron-phonon interaction, on a much longer time scale, which leads to the final, full melting of the PLD. For $\mathrm{LaSr}_{2} \mathrm{Mn}_{2} \mathrm{O}_{7}$ our time-resolved electron diffraction study reveals the dynamic path of atoms and ions and indicates that Jahn-Teller-like $\mathrm{O}, \mathrm{La} / \mathrm{Sr}$, and $\mathrm{Mn} 4+$ displacements dominate the lattice response and exhibit a dichotomy in behavior - overshoot-and-recovery for one sublattice versus normal behavior for the other. This dichotomy, attributed to slow electronic relaxation, proves that polaron transport is a key process in doped manganites. Our study shed light on the various and often intertwined degrees of freedom and their responses to external perturbations in strongly correlated electron systems [6].

\section{References:}

[1] D.J. Flannigan et al, PNAS, 1079933 (2010).

[2] J.S. Kim et al, Science 3211472 (2008).

[3] M. Eichberger et al, Nature 468, 799 (2010)

[4] P. Zhu et al, Appl. Phys. Lett., 103071914 (2013).

[5] P. Zhu et al, "Femtosecond Time-resolved MeV Electron Diffraction”, New J. of Physics, 17063004 (2015). 
[6] The authors would like to thank S.W. Cheong, J. Cao, Y. Hidaka, J. Geck, S. Pjerov, T. Ritschel, H.Berger, Y.Shen, and R.Tobey for providing samples and assistance. Work supported by the U.S. DOE, under contract No. DE-SC0012704.

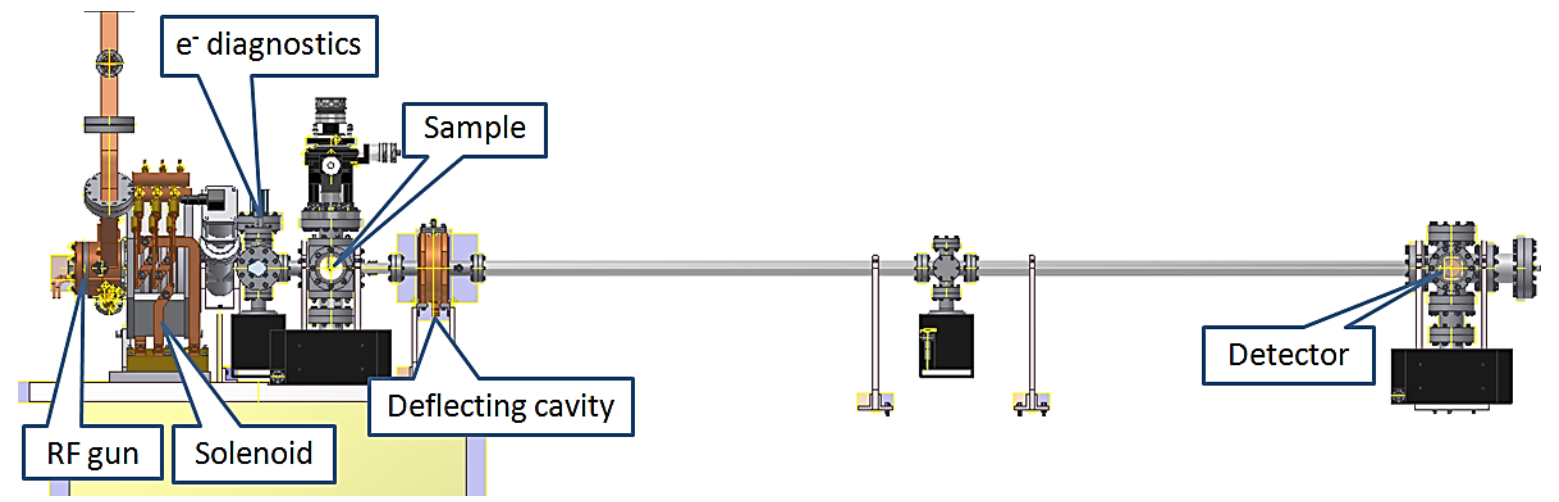

Fig. 1. Schematic of the relativistic MeV-UED (ultrafast electron diffraction) set-up at Brookhaven national Laboratory. The entire system is $\sim 4.5 \mathrm{~m}$ long. UV photons from a Ti-sapphire laser are used to generate electrons in the RF gun. A solenoid magnet focuses the beam onto the detector screen $4 \mathrm{~m}$ downstream of the sample. NearIR pulses from the same Ti-sapphire laser are used to optically pump the sample. The instrument has a cryogenic capability and can operates at $2-4 \mathrm{MeV}$ with $10^{6}$ electron per pulse to achieve $120 \mathrm{fs}$ temporal resolution.
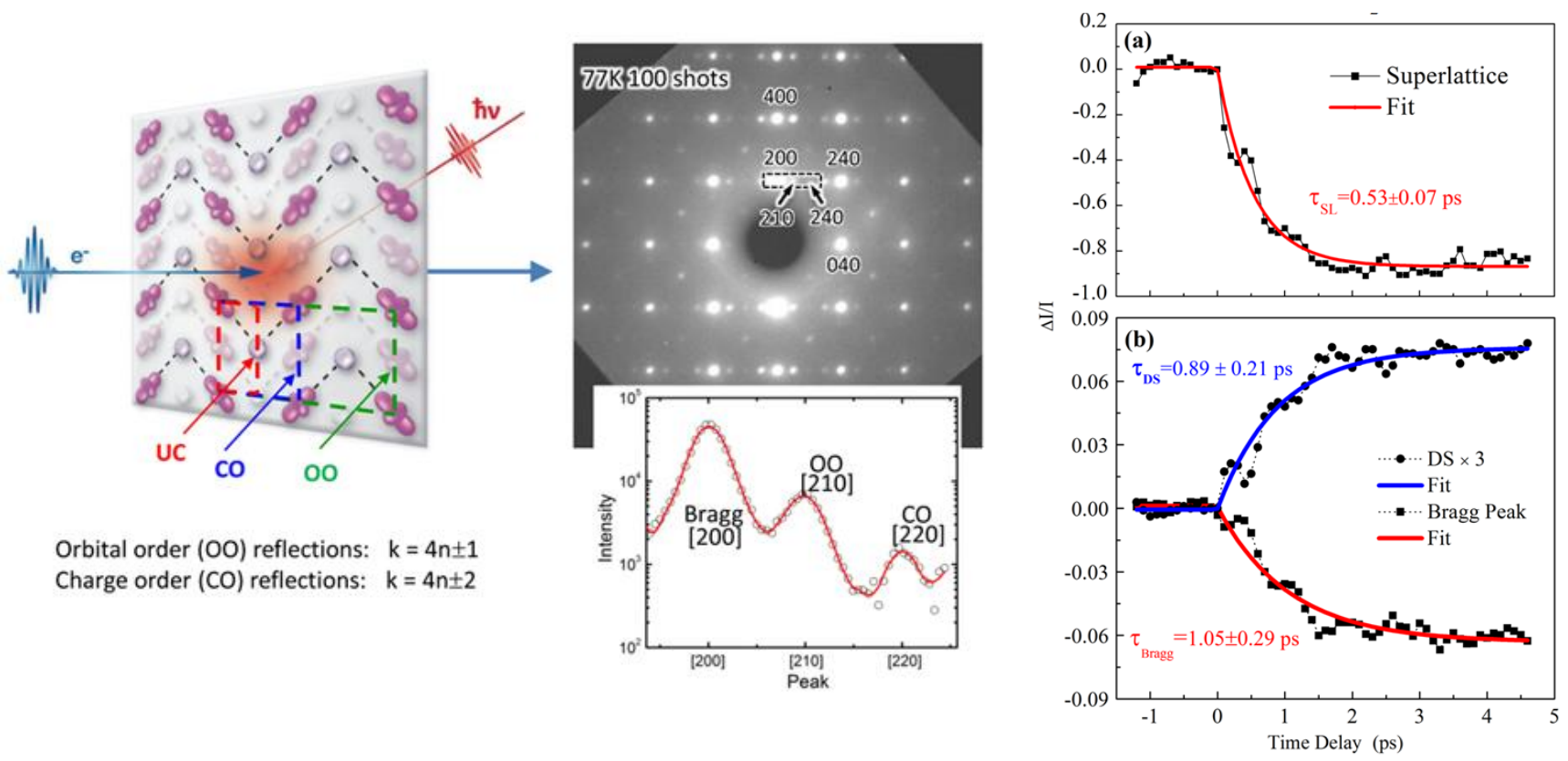

Fig. 2 (left): Geometrical arrangement of pump and probe in the MeV-UED experiment. A $800 \mathrm{~nm}$ laser with a duration of $150 \mathrm{fs}$ is used to pump $\mathrm{LaSr}_{2} \mathrm{Mn}_{2} \mathrm{O}_{7}$ single crystal. (middle): A typical diffraction pattern of LSMO with integrated intensity profile from the boxed area for quantitative analysis. (right): Temporal evolution of the charge density wave (CDW) superlattice peak (a) and the Bragg peak and diffuse scattering intensity (b) in $2 \mathrm{H}-$ $\mathrm{TaSe}_{2}$. For clarity, the change of diffuse scattering intensity is multiplied by 3 . The pump fluence was $1.4 \mathrm{~mJ} / \mathrm{cm}^{2}$. The solid lines with given time constants are fits to the experimental data using an exponential function. 